\title{
El desarrollo regional y la sostenibilidad: revisión sistemática y análisis bibliométrico
}

\author{
Julio César Silva Rincón* \\ Jhon Antuny Pabón León** \\ Ender José Barrientos Monsalve ${ }^{\star \star \star}$
}

Fecha de recibido: 19 de marzo de 2021

Fecha de aprobado: 29 de septiembre de 2021

Para citar este artículo: Silva Rincón, J. C., Pabón León, J. A., \& Barrientos Monsalve, E. J. (2021). El desarrollo regional y la sostenibilidad: revisión sistemática y análisis bibliométrico. Revista Universidad \& Empresa, 23(41), 1-36. https://doi.org/10.12804/

revistas.urosario.edu.co/empresa/a.10403

\section{Resumen}

El propósito de este trabajo es realizar una revisión sistemática y análisis bibliométrico de publicaciones sobre el desarrollo regional y la sostenibilidad, dos conceptos cada vez más importantes en los foros económicos, ambientales, sociales y académicos de todos los países del mundo. Se realiza bajo el concepto de cartografía científica, utilizando la bibliometría para observar las interrelaciones entre disciplinas, campos, profesiones o documentos. Para esto, se desarrollaron dos etapas: análisis bibliométrico y análisis de redes. Primero, se llevó a cabo una búsqueda WoS y se revisaron los indicadores bibliométricos. En el

* Administrador de Empresas, MSc (e) Gerencia de Empresas. Gobernación de Norte de Santander. Correo electrónico: j.silva@nortedesantander.gov.co ORCID: https://orcid.org/0000-0003-3515-1039

** Administrador de Empresas, MSc Gerencia de Empresas, Universidad Francisco de Paula Santander. Correo electrónico: jhonantuny@ufps.edu.co ORCID: https://orcid.org/0000-0002-1078-2857

*** Doctor en Ciencias Gerenciales, Fundación de Estudios Superiores Comfanorte. Correo electrónico: ej_barrientos@fesc. edu.co ORCID: https://orcid.org/0000-0001-6673-0223 
segundo paso, con la información y las referencias bibliográficas del primer paso, se construye la red a través del análisis de las cocitaciones, comparado con la similitud del árbol. Estos documentos se dividen en tres categorías: la raíz del documento fuente (denominados hegemónicos o clásicos); la columna vertebral del documento involucrado en el desarrollo del conocimiento actual de la materia (denominados tronco o estructurales); y la recopilación de las últimas novedades (denominadas hojas o perspectivas de trabajo del documento). Finalmente, se presentan tres perspectivas identificadas en los siguientes campos de la investigación: planeación y desarrollo territorial; evaluación y ecosistemas; China y el urbanismo.

Palabras clave: desarrollo regional; ecosistemas; urbanismo; mapeo científico.

\title{
Regional Development and Sustainability: Systematic Review and Bibliometric Analysis
}

\begin{abstract}
The purpose of this work is to perform a systematic review and a bibliometric analysis of publications on regional development and sustainability, two increasingly important concepts in economic, environmental, social, and academic forums in all world countries. The study was carried out under the concept of scientific mapping, using bibliometrics to observe the interrelations between disciplines, fields, professions, or documents. The study was done in two stages: a bibliometric analysis and a network analysis. First, a WoS search was performed, and bibliometric indicators were reviewed. In the second step, the network was constructed through the analysis of co-citations, which were compared with the similarity tree. These documents are divided into three categories: the root of the source document (called hegemonic or classic); the backbone of the document involved in the development of the current knowledge of the subject called trunk or structural; the last one is the collection of the latest developments called leaves or perspectives of the document work. Finally, three perspectives identified in the following research fields are presented: planning and territorial development, evaluation and ecosystems, and China and urban development.
\end{abstract}

Keywords: Ecosystems; scientific mapping; regional development; urbanism.

\section{Desenvolvimento regional e sustentabilidade: revisão sistemática e análise bibliométrica}

\section{Resumo}

O objetivo deste trabalho é realizar uma revisão sistemática e análise bibliométrica de publicações sobre desenvolvimento regional e sustentabilidade, dois conceitos cada vez mais importantes em fóruns econômicos, ambientais, sociais e acadêmicos em todos os países do mundo. É realizado sob o conceito de cartografia científica, utilizando a bibliometria para observar as inter-relações entre disciplinas, campos, profissões ou documentos. Para isso, foram desenvolvidas duas etapas: análise bibliométrica e análise de redes. Primeiramente, uma pesquisa WoS foi realizada e indicadores bibliométricos foram revisados. Na segunda etapa, com as informações e referências bibliográficas da primeira etapa, a rede é construída por meio da análise das cocitações, comparada com a semelhança a uma árvore. Esses documentos são divididos em três categorias: a raiz do documento fonte (denominada hegemônica e/ou clássicas); a espinha dorsal do documento envolvida no desenvolvimento do conhecimento atual do assunto denominado tronco e/ou estruturais; e a última é a compilação das últimas novidades denominadas folhas ou perspectivas de trabalho do documento. Por fim, são apresentadas três perspectivas identificadas nos seguintes campos de pesquisa: planejamento e desenvolvimento territorial; avaliação e ecossistemas; China e o Urbanismo.

Palavras-chave: desenvolvimento regional; ecosistemas; urbanismo; mapeamento científico. 


\section{Introducción}

El desarrollo regional y la sostenibilidad son dos conceptos de gran relevancia en los foros económicos, ambientales, sociales y académicos de los países del mundo. Diversas publicaciones han permitido ahondar en los anteriores enunciados, generando expectativas para la producción y conservación sostenible. Secundo et al. (2020) proporcionan una revisión estructurada de la literatura sobre el papel estratégico del capital intelectual para el cumplimiento de los Objetivos de Desarrollo Sostenible.

Por otro lado, Choi y Labhsetwar (2020) mencionan que las estrategias de producción nacional de alimentos y del uso del agua en una sociedad cambiante variarán en función del desarrollo rural y económico. Por su parte, Stoffelen (2020) critica la postura generalizada de los estudios sobre los parques geológicos al aceptar y validar casi unánimemente el valor de estos como instituciones de protección del paisaje y como impulsores del desarrollo regional sostenible, sin comprometerse críticamente con los procesos sociales que, de hecho, determinan si se pueden hacer esas afirmaciones.

Balaban et al. (2019) afirman que la falta de conceptos de sostenibilidad conduce a un uso irrazonable de recursos y energía, creándose un desequilibrio a largo plazo entre insumos y productos. Kim y Lim (2017) aportan conocimiento en cuanto al papel de las organizaciones en la economía social, haciendo un estudio de estas con un enfoque en las empresas sociales y sus vínculos teóricos con la sostenibilidad.

Se han efectuado otras investigaciones que permiten complementar conocimiento y dar herramientas para abordar el desarrollo regional de manera sostenible. Tenemos que Cohn et al. (2017) compararon los sistemas agrícolas a pequeña escala con otros sistemas agrícolas en el clima cambiante y establecieron fuertes contrastes y una topografía imprecisa. Geneletti et al. (2017) plantearon mejor la relación entre la planificación sostenible y los diferentes tipos de áreas urbanas circundantes, seleccionaron lecciones de las aplicaciones existentes y formularon los desafíos para el desarrollo futuro de la investigación en planificación. 
Rinkinen et al. (2016) aportaron a la teoría del desarrollo regional al incorporar el concepto de sostenibilidad al debate sobre la teoría del desarrollo. Rinkinen et al. (2016) y Russo et al. (2014) valoraron el cuidado de los bienes hídricos en tres sectores: las ciudades, la agricultura y los sistemas naturales. Servillo et al. (2012) contribuyeron a la definición de "atractivo territorial" desde el enfoque abstracto y en términos de política, como también a cooperar a la creación de una base más sistematizada para la integración de las estrategias de desarrollo regional en la política espacial. Sin embargo, las investigaciones anteriores han encontrado lagunas de conocimiento, proporcionando una vía para los estudios que se requiere complementar en el futuro para el desarrollo regional y la sostenibilidad.

A partir de los análisis anteriores, se hace necesario realizar una revisión que permita identificar las corrientes o perspectivas en las que se enmarca la investigación en el área en la actualidad. Por ejemplo, Choi y Labhsetwar (2020) proponen que, para resolver el problema de la inseguridad alimentaria, es preciso efectuar otras investigaciones o trabajos de investigación a fin de encontrar formas efectivas de utilizar los recursos hídricos para la producción agrícola y los servicios ambientales, al tiempo que se logra un desarrollo rural sostenible.

Así mismo, Secundo et al. (2020) consideran que se requiere complementar investigaciones en áreas de: componentes del capital intelectual para la sostenibilidad del desarrollo regional sostenible en el sector privado, en la economía del conocimiento; y en el sector público. Igualmente, es fundamental avanzar en estudios o investigaciones en el campo de la importancia de la tecnología para abordar los Objetivos de Desarrollo Sostenible. Por otro lado, Stoffelen (2020) presenta la necesidad explícita de ir más allá del ámbito de las geociencias para crear nuevas y significativas percepciones del papel social de los parques geológicos.

Se trata de buscar una vía intermedia para que las interacciones entre el hombre y el medio ambiente, en este caso en el contexto de los parques geológicos, puedan analizarse desde distintos ángulos y combinarse en un enfoque transdisciplinario. Para Rinkinen et al. (2016), es necesario explorar más a fondo cómo se producen estas transformaciones sociales que tienen como objetivo el desarrollo sostenible, cómo pueden ser apoyadas por diversos medios políticos y qué opciones requiere la sociedad para tales transformaciones. 
Considerando la relevancia de este tema, se busca revisar la literatura científica sobre desarrollo regional y sostenibilidad a través de este trabajo, enfocándose en las siguientes preguntas: ¿cuáles son los autores, revistas, países e instituciones más influyentes en literatura sobre el tema de desarrollo regional y sostenibilidad? ¿Cuál es el enfoque o línea en la que se desarrolla en la actualidad el desarrollo regional y sostenibilidad?

Este artículo se divide en cuatro partes. La primera es el método detallado, que explica la elección de la información y las herramientas utilizadas. Luego, se propone y menciona un análisis estadístico de los resultados de cada consulta, acompañado de un análisis bibliométrico del año, revista, autor, organización, país y autor más citado. Posteriormente, se introduce la red y análisis de clasificación, coocurrencia de palabras, colaboración entre autores y colaboración entre países y tendencias; este último es el resultado del análisis de conglomerados. Finalmente, se mencionan las conclusiones, limitaciones y sugerencias para futuras investigaciones.

\section{Metodología}

El estudio de la investigación se adelantó bajo el concepto del método del mapeo científico, usando métodos bibliométricos para observar cómo los documentos de diferentes disciplinas, campos, especialidades o individuos están relacionados entre sí (Zupic \& Čater, 2015). Para ello se llevaron a cabo dos etapas: análisis bibliométrico y análisis de red. Primero, se efectuó la búsqueda en WoS, revisando los indicadores bibliométricos; y en el segundo, ya con la información referenciada en el primer paso y sus referencias bibliográficas, se levantó la red con $\mathrm{R}$ a través del análisis de cocitaciones, asimilando la analogía del árbol —siglo III a. C. por Porphyry, según Hughes (2010)—; recientemente por Gentner (1983) y Levy (2002), identificando las perspectivas de investigación y visualizándolas con Gephi. 


\subsection{Análisis bibliométrico (etapa 1)}

A partir de las limitaciones y sugerencias de revisiones anteriores se utilizó WoS para buscar documentos bajo los siguientes parámetros (con su respectiva ecuación de búsqueda en la tabla 1).

Tabla 1. Criterio de búsqueda y resultados

\begin{tabular}{ll}
\hline Bases de datos & Web of Science \\
Espacio de tiempo & $2000-2019$ \\
Fecha de consulta & 18 de octubre de 2020 \\
Tipo de document & Article, books, book chapters and conference papers \\
Tipo de revista & All types \\
Campo de búsqueda & Title, abstract, keywords \\
Términos de búsqueda & Tema: 'regional development' y 'sustainable development' \\
Resultados generales & 756 \\
\hline
\end{tabular}

Fuente: elaboración de los autores (2020).

Con los términos de investigación se obtuvo una producción de 756 documentos en WoS, empleando un enfoque cuantitativo. Para el análisis del desempeño y el mapeo científico, se utilizan cinco métodos bibliométricos (Zupic \& Čater, 2015): análisis de citas, análisis de las palabras clave, análisis de la cocitación, análisis del coautor, análisis de acoplamiento bibliográfico.

El primero señala el histórico de artículos, clasificados por base de datos, países, revistas y autores. El segundo detecta las palabras más frecuentes que se encuentran presentes en las palabras clave de todos los artículos. El tercero muestra la red de cocitaciones y colaboración. El cuarto indica la red de coautorías, en la que se representa el apoyo entre los autores. El quinto enlaza los documentos teniendo en cuenta las referencias compartidas, logrando determinar los campos emergentes, es decir, las perspectivas (red).

\subsection{Análisis de red (etapa 2)}

Para el levantamiento de la red se usó la teoría de grafos, que deja ver los documentos y la forma en que se enlazan. Este método permite generar información asociada a la tipología y características de la red y de cada documento que la conforma (Wallis, 2007; Wasserman 
\& Faust, 1994; Yang et al., 2016). Así, los registros alcanzados se fusionan, se extrae sus referencias (bibliografía) eliminando copias y se construye una red de intercitaciones mediante programación en $\mathrm{R}$.

Para observar e interpretar la red, se emplea Gephi de Mathieu et al. (2009) en concordancia con los estudios previos (Donato, 2017; Donthu et al., 2020; Ferguson, 2012; Jacomy et al., 2014; Meier, 2020). Después, se calculan el indegree —cantidad de veces que un documento ha sido mencionado por otros, según Wallis (2007)—; el outdegree —cantidad de veces que un nodo en particular referencia a otros o el número de conexiones de cada documento, según Wallis (2007)—; y el betweenness — nivel de intermediación y centralidad del elemento dentro de la red, según Freeman (1977)—, permitiendo ver cuándo el documento referencia ha sido citado por los demás (Zhang \& Luo, 2017).

La red aflora de las referencias extraídas de los artículos, calculando indegree, outdegree y betweenness, para generar el esquema propuesto por Robledo et al. (2014), acogiendo la analogía del árbol. Se ordenan los documentos en tres categorías: las raíces (alto indegree), donde se localizan los documentos fuente, hegemónicos, clásicos o la base intelectual de conocimiento de un campo (Persson, 1994), que son citados pero no citan a otros; el tronco (alto betweenness), que involucra los documentos que generan las aplicaciones fuertes en el desarrollo del conocimiento actual del tema o concepto examinado, estos citan y a la vez son citados por otros, y, por lo tanto, son conocidos como estructurales o estructura intelectual, que marca las líneas de investigación en el campo, por lo que, además de mostrar su composición, presenta los temas de investigación más importantes en la literatura y el modelo de sus interrelaciones (Shafique, 2013); finalmente, las hojas (alto outdegree), las cuales reúnen los documentos más recientes que citan a los demás en la base de conocimiento (tronco y raíces) pero no son anotados, mostrando los frentes de investigación (Price, 1965) y visibilizando las perspectivas. Esta forma de trabajo ha sido empleada en otros estudios (Buitrago et al., 2020; Díez-Gómez et al., 2019; Duque et al., 2021; Duque, Samboni, et al., 2020; Duque, Toro, et al., 2020; Duque \& Cervantes-Cervantes, 2019; Duque \& Duque, 2020; Salazar et al., 2020).

Para identificar a partir de la red de referencias los clústeres o subáreas del tema, se utilizó el algoritmo de clusterización presentado por Blondel et al. (2008). Este procedimiento permite a través de un estudio de cocitaciones clasificar los documentos en las categorías 
establecidas. Posteriormente, mediante minería de texto programada en $\mathrm{R}$ con el paquete WordCloud (Ohri, 2012), se identifican los temas que componen los clústeres. Una vez identificadas dichas perspectivas bajo criterios bibliométricos (Zupic \& Čater, 2015), se revisa la literatura seleccionando los 50 documentos más importantes, así: 10 de la raíz (clásicos), 10 del tronco (estructurales) y 30 de las hojas (perspectivas bibliométricas). A manera de ejemplo de la revisión de los documentos, se describen los cuatro más relevantes (por número de referencias dentro de la red), por lo cual se consideran hegemónicos.

Finalmente, a partir de los clústeres identificados, se estructuraron subredes con los documentos correspondientes y se generaron nubes de palabras, las cuales se comparan con la lectura de los documentos para así identificar las temáticas abordadas en cada grupo; además, para considerar las conveniencias de investigación.

\section{Desarrollo de la investigación Número de publicaciones por año}

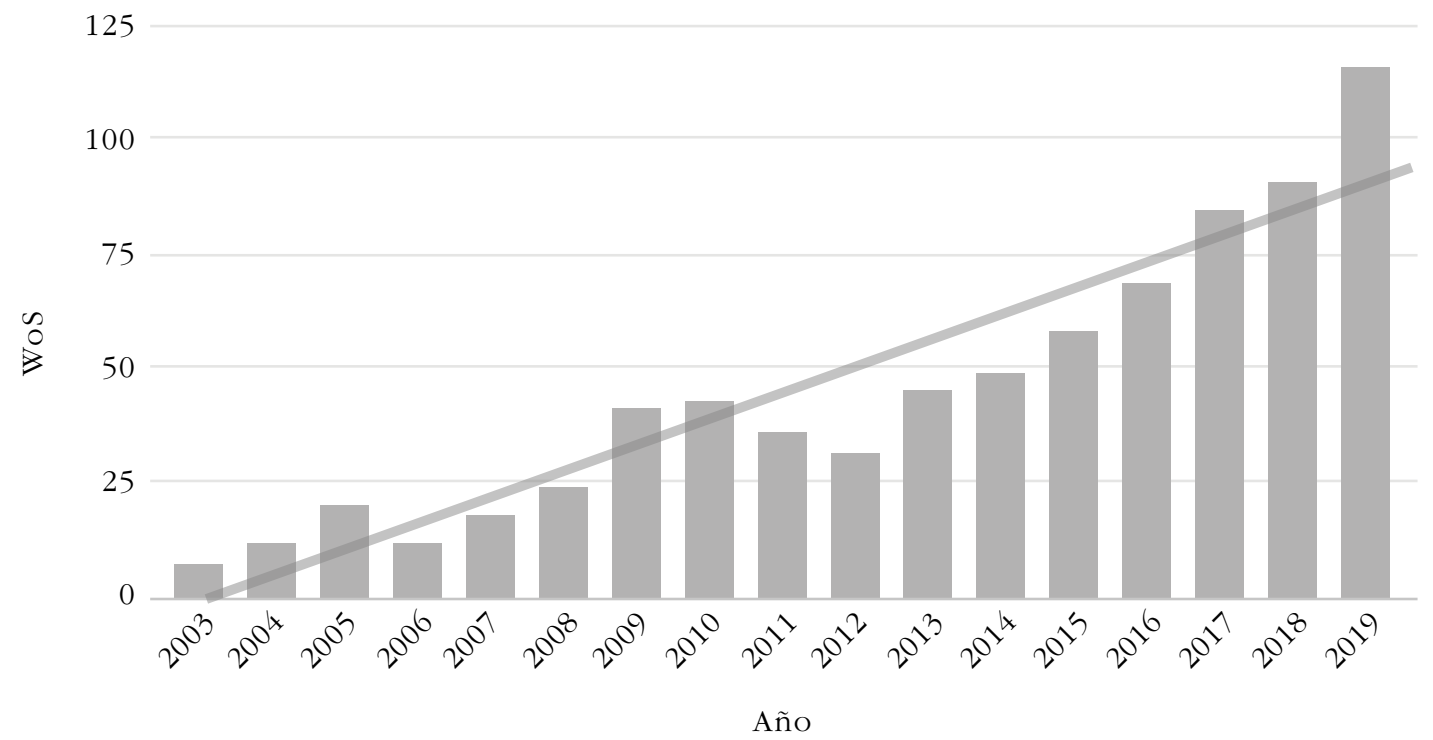

Figura 1. Número de publicaciones por año Fuente: elaboración de los autores. 
Al analizar las tendencias y variación del número de publicaciones por año y comparando las veces de publicaciones registradas en Web of Science desde 2003 hasta 2019, se han sumado un total de 756 artículos. Como muestra esta línea, la inclinación de la sociedad científica por este campo del conocimiento ha aumentado, reflejando una tasa de crecimiento anual del $17.39 \%$, y la producción científica en los últimos cinco años representó el $52.53 \%$ del total de publicaciones.

Adicionalmente, es claro que estas publicaciones comenzaron en 2003 con 7 artículos al inicio, y, para 2019, esta cifra ha aparecido en 756 artículos. El aumento significativo fue a partir de 2019 a 2020, de 91 a 116, respectivamente, coincidiendo con la adopción de los 17 objetivos de desarrollo sostenible por parte de la cumbre de las Naciones Unidas, reunidas en septiembre del año 2020, en Nueva York.

\section{Fuente de publicaciones}

Tabla 2. Fuente de publicaciones

\begin{tabular}{|c|c|c|c|c|c|c|}
\hline Publicaciones & WoS & $\begin{array}{l}\text { \% del } \\
\text { total }\end{array}$ & SJR 2019 & Cuartil & $\begin{array}{l}\text { Índice } \mathrm{H} \\
\text { (sJR) }\end{array}$ & País \\
\hline Sustainability & 48 & $6.35 \%$ & 0.58 & Q2 (SJR) & 68 & Suiza \\
\hline Journal of Cleaner Production & 18 & $2.38 \%$ & 1.89 & Q1 (SJR) & 173 & $\begin{array}{l}\text { Países } \\
\text { Bajos }\end{array}$ \\
\hline Economic Science for Rural Development & 11 & $1.46 \%$ & - & - & - & - \\
\hline $\begin{array}{l}\text { International Multidisciplinary Scientific } \\
\text { Conferences on Social Sciences and Arts }\end{array}$ & 8 & $1.06 \%$ & - & - & - & - \\
\hline $\begin{array}{l}\text { Medunarodni Znanstveni Simpozij Gospodarstvo } \\
\text { Istocne Hrvatske Jucer Danas Sutra }\end{array}$ & 8 & $1.06 \%$ & - & - & - & - \\
\hline $\begin{array}{l}\text { Wit Transactions on Ecology and The } \\
\text { Environment }\end{array}$ & 8 & $1.06 \%$ & 0.14 & Q4 (SJR) & 20 & $\begin{array}{l}\text { Reino } \\
\text { Unido }\end{array}$ \\
\hline European Planning Studies & 7 & $0.93 \%$ & 0.95 & Q1 (SJR) & 75 & $\begin{array}{l}\text { Reino } \\
\text { Unido }\end{array}$ \\
\hline International Multidisciplinary Scientific & 7 & $0.93 \%$ & 0.23 & $\mathrm{Nr}$ & 17 & Bulgaria \\
\hline \multicolumn{7}{|l|}{ Geoconference SGEM } \\
\hline Baltic Journal of Economic Studies & 6 & $0.79 \%$ & - & - & - & - \\
\hline Ecological Indicators & 6 & $0.79 \%$ & 1.33 & Q1 (SJR) & 113 & $\begin{array}{l}\text { Países } \\
\text { Bajos }\end{array}$ \\
\hline
\end{tabular}

Fuente: elaboración de los autores (2020). 
En esta sección se muestra cómo se distribuyen las publicaciones entre diferentes revistas e indica el número de registros obtenidos en WoS. Para ello, es necesario considerar algunas variables, como el indicador SJR (revista scImago y ranking de países/regiones), que se utiliza para medir la influencia científica del artículo promedio en la revista. El cuartil para clasificarlos según JCR (Journal Citation Report). Finalmente, el índice h se ha registrado en SJR.

En la tabla 2 se observan las diez revistas con mayor cantidad de publicaciones sobre este tema, que representan el $16.81 \%$ con relación a los 756 artículos publicados. La revista con mayor producción es Sustainability, que representa el 6.35\% de los documentos. Tres revistas no permitieron reconocer el indicador SJR ni el cuartil para clasificarlas según JCR, tampoco el índice h se ha registrado en sJR. Suiza, Holanda, Inglaterra y Bulgaria son los países donde se generó la publicación de las revistas identificadas.

\section{Análisis de autores y coautores}

Tabla 3. Mejores diez autores

\begin{tabular}{lccc}
\hline \multicolumn{1}{c}{ Autor } & $\begin{array}{c}\text { Número de } \\
\text { publicaciones }\end{array}$ & $\begin{array}{c}\text { WoS } \\
\text { Número de citaciones }\end{array}$ & Índice $\mathbf{h}$ \\
\hline Fan, Jie & 5 & 891 & 17 \\
\hline Adamisin, Peter & 4 & 151 & 8 \\
\hline Conradin, Katharina & 4 & 24 & 3 \\
\hline Gibbs, David & 4 & 2612 & 28 \\
\hline Liu, Yi & 4 & 372 & 10 \\
\hline Lu, Zhaohua & 4 & 779 & 15 \\
\hline Muresan, Mihaela & 4 & 710 & 12 \\
\hline Tan, Feifei & 4 & 1091 & 20 \\
\hline Wang, Lei & 4 & 899 & 17 \\
\hline Apanaviciene, Rasa & 3 & 51 & 4 \\
\hline
\end{tabular}

Fuente: elaboración de los autores (2020).

La tabla 3 enumera los diez autores que han trabajado más en este tema. Los indicadores incluyen: el número de citas y el índice —el índice publicado por Hirsch (2005) para medir la productividad científica de cada investigador-. Además, se mencionan todas las publicaciones de cada autor. 
Según la información obtenida en WoS entre 2000 y 2019, el autor con mayor publicación es Jie Fan, alcanzando 5 publicaciones, con 891 citaciones. Por otro lado, el autor más citado es David Gibbs, con 2612 citaciones y 4 publicaciones; seguido por Feifei Tan, con 1091 citaciones con 4 publicaciones; y Ley Wang, con 899 citaciones e igual número de publicaciones.

\section{Análisis de afiliaciones}

Tabla 4. Análisis de afiliaciones

\begin{tabular}{lcc}
\hline \multicolumn{1}{c}{ Organización } & Número de artículos & País \\
\hline Chinese Academy of Sciences & 36 & China \\
\hline University of the Chinese Academy of Sciences & 18 & China \\
\hline Vilnius Gediminas Technical University & 13 & Lituania \\
\hline Bucharest Academy of Economic Studies & 10 & Rumania \\
\hline China University of Geosciences & 10 & China \\
\hline Beijing Normal University & 9 & China \\
\hline Wageningen University & 9 & Países Bajos \\
\hline University of Mining and Technology in China & 7 & China \\
\hline Wuhan University & 7 & China \\
\hline Kaunas University of Technology & 6 & Lituania \\
\hline
\end{tabular}

Fuente: elaboración de los autores (2020).

La tabla 4 enumera las diez organizaciones con el mayor número de producción científica registrada en este estudio bibliométrico. La Chinese Academy Sciences contribuye con 36 artículos, seguida por la University of the Chinese Academy of Sciences, con 18 artículos. Las diez universidades aportan 125 artículos de los 756 artículos del análisis. En China se encuentran 6 universidades de las 10 de la muestra. 


\section{Análisis por países/regiones}

Tabla 5. Mejores diez países/regiones

\begin{tabular}{lcc}
\hline \multicolumn{1}{c}{ País/región } & $\begin{array}{c}\text { WoS } \\
\text { Número de artículos }\end{array}$ & $\begin{array}{c}\% \text { del } \\
\text { total }\end{array}$ \\
\hline República Popular de China & 170 & $22.5 \%$ \\
\hline Rumania & 61 & $8.1 \%$ \\
\hline Rusia & 53 & $7.0 \%$ \\
\hline Polonia & 37 & $4.9 \%$ \\
\hline Estados Unidos & 37 & $4.9 \%$ \\
\hline Alemania & 31 & $4.1 \%$ \\
\hline Eslovaquia & 28 & $3.7 \%$ \\
\hline Ucrania & 27 & $3.6 \%$ \\
\hline Lituania & 26 & $3.4 \%$ \\
\hline Países Bajos & 26 & $3.4 \%$
\end{tabular}

Fuente: elaboración de los autores (2020).

De acuerdo con WoS, la tabla 5 enumera los diez países/regiones con el mayor número de publicaciones sobre este tema. China muestra el $22.5 \%$ de toda la información analizada. En segundo y tercer lugar se encuentran Rumania y Rusia, con el $8.1 \%$ y el 7.0\% de la producción científica mundial, respectivamente.

\section{Mejores diez documentos más citados}

Tabla 6. Diez documentos más citados

\begin{tabular}{lcc}
\hline \multirow{2}{*}{ Autores, años } & \multicolumn{2}{c}{ Citación global } \\
\cline { 2 - 3 } & WoS & Google Scholar \\
\hline Zavadskas y Turskis (2011) & 357 & 566 \\
\hline Lawhon y Murphy (2012) & 225 & 489 \\
\hline While et al. (2010) & 213 & 406 \\
\hline Mirata y Emtairah (2005) & 143 & 367 \\
\hline Del Río y Burguillo (2008) & 120 & 311 \\
\hline Del Río y Burguillo (2009) & 98 & 233 \\
\hline Fan (2006) & 98 & 251 \\
\hline Wei y Ye (2014) & 95 & 127 \\
\hline Jha y Bawa (2006) & 91 & 194 \\
\hline
\end{tabular}

Fuente: elaboración de los autores (2020). 
La tabla 6 enumera los diez documentos más citados. La forma más común de determinar la relevancia de un artículo es el número de citas recibidas (Ding \& Cronin, 2011). En otras palabras, cada artículo está relacionado con las citas que recibe dentro de la red de 'citas locales' y fuera de la red de 'citas globales'. La primera representa el número de veces que el artículo ha sido citado por otros en WoS. La segunda columna muestra la cantidad de veces que se ha citado el trabajo en Google Scholar.

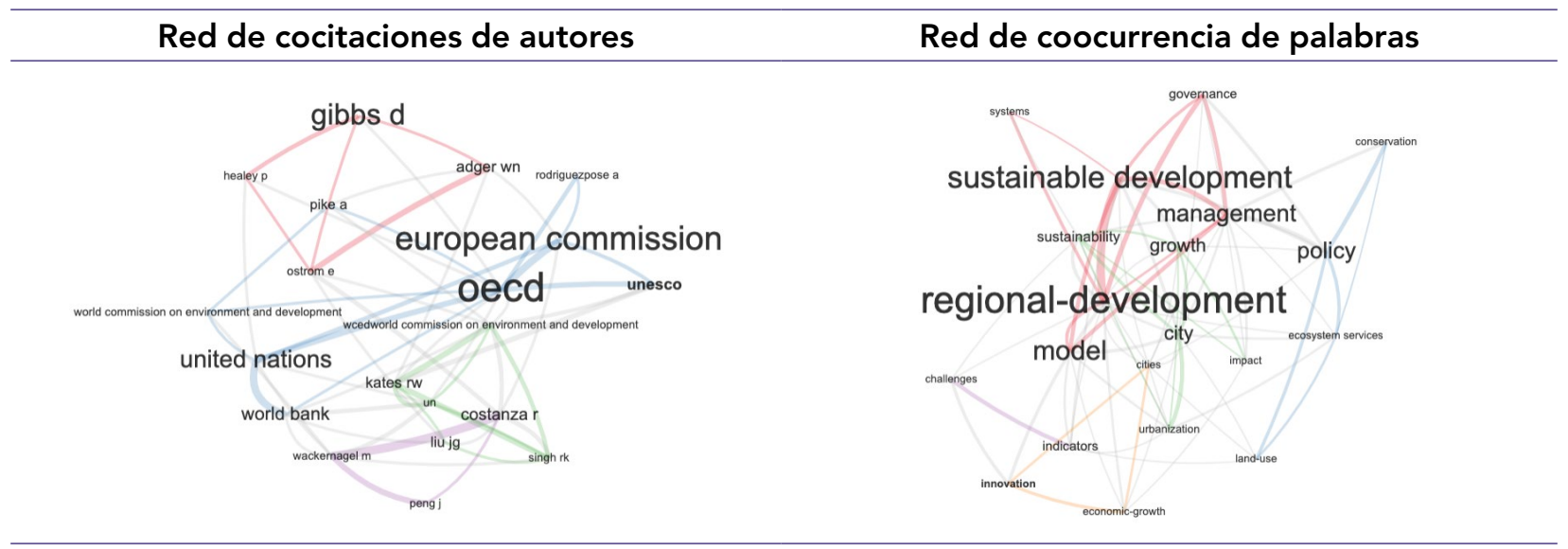

Figura 2. Red de cocitaciones de autores y coocurrencia de palabras

Fuente: elaboración de los autores (2020).

En la figura 2 se ilustra la red de cocitaciones de autores que representa el número de autores que resultan más referenciados en la base de datos. Como se aprecia, sobresalen y predominan 20 autores, siendo la European Commission el autor más citado, luego aparece la oECD y otros como D. Gibbs, United Nations, World Bank, UnEsCo y R. Costanza.

Igualmente, la figura muestra una red de coocurrencia de palabras. Es decir, la interconexión de palabras relacionadas. Proviene de las palabras clave de cada artículo que conforma la red. Para este caso, se seleccionaron las 20 veces más repetidas. La nube de palabras hace referencia a las palabras más repetidas en las publicaciones, como región, desarrollo, sostenibilidad, modelo, gestión, política, sostenibilidad, crecimiento, ciudad. 


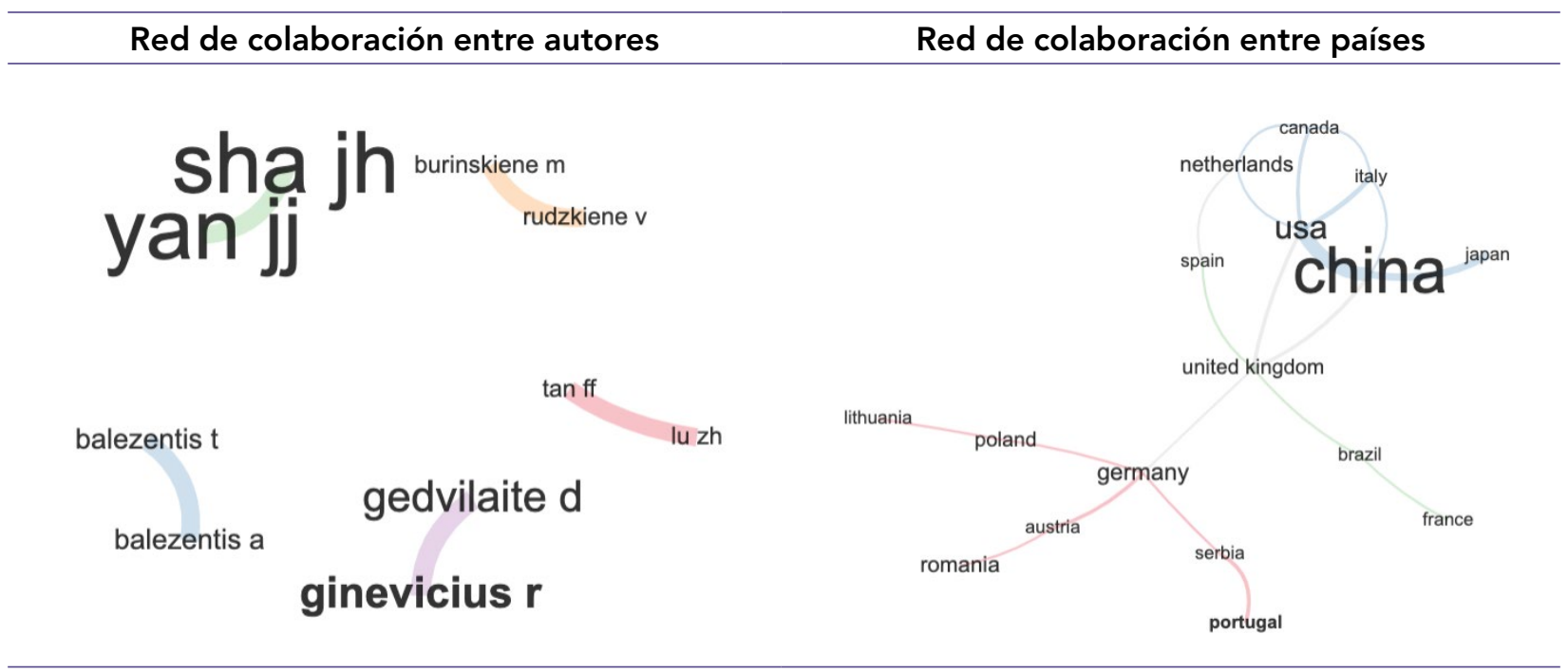

Figura 3. Red de colaboración entre autores y de colaboración entre países

Fuente: elaboración de los autores (2020).

La figura 3 expone la red de colaboración entre autores. Para ello, se tomó como referencia el período comprendido entre el año 2000 y 2019, aplicando la herramienta Bibliometrix para registros generados mediante consultas WoS. Cada nodo está representado por un autor, del cual se seleccionan nodos (coautores) con más de 50 publicaciones y como mínimo tres conexiones, dando como resultado una red de nueve investigadores. El tamaño del nombre del autor representa el número de artículos. La relación de colaboración entre investigadores se manifiesta en las rutas que los conectan, y su profundidad significa más trabajos de coautoría. Se encontraron cinco grupos de colaboración que cumplían con los criterios anteriores. El grupo principal está compuesto por Shaji y Yan Jijie.

De igual manera, la figura da cuenta de una red de cooperación entre países, mostrando 17 opciones de contacto entre países, y la respuesta es una fuerte cooperación entre tres grupos. El grupo principal está compuesto por China, Estados Unidos y Japón; refleja una autoría más transnacional. Este grupo se destaca porque está compuesto por países de dos continentes diferentes, América y Asia. 


\section{Mapa de cocitaciones}

Un mapa de cocitaciones es la ilustración gráfica de los artículos que integran la red, conformados por nodos y un grupo de aristas que representan su concurrencia. Este mapa se basa en la teoría de grafos, técnica que permite emanar información asociada al tipo y características de la red y de cada uno de los documentos que la componen (Wasserman \& Faust, 1994; Yang et al., 2016).

En el mapa de cocitaciones, se encuentran áreas de alta densidad, las cuales reúnen información que se referencia entre sí, indicando la existencia de aspectos en común de investigaciones (Braam et al., 1991). Además, un gran número de citación entre los documentos implica una alta probabilidad de que estén relacionados, siendo un indicio de que pertenecen a un área de estudio en común (Hjørland, 2013).

Para poder identificar en el mapa de cocitaciones los campos de estudio en común de investigación (grupo de documentos cocitados), se usó el algoritmo de clusterización dado por Blondel et al. (2008), instrumento que permite, a través de un estudio de citas, organizar la información en clústeres o categorías de diferentes colores. Seguidamente se utiliza el algoritmo Force Atlas 2 (Jacomy et al., 2014), ubicando los nodos que más se conectan con el centro del mapa, mientras que los que menos se conectan son desplazados a los bordes, evitando su solapamiento. Por último, se emplea Gephi para la visualización de la red relacionada con el desarrollo regional y la sostenibilidad, aplicando algoritmos que permitieron la identificación de tres clústeres principales en esta área de estudio, ilustrados en la figura 4 . 


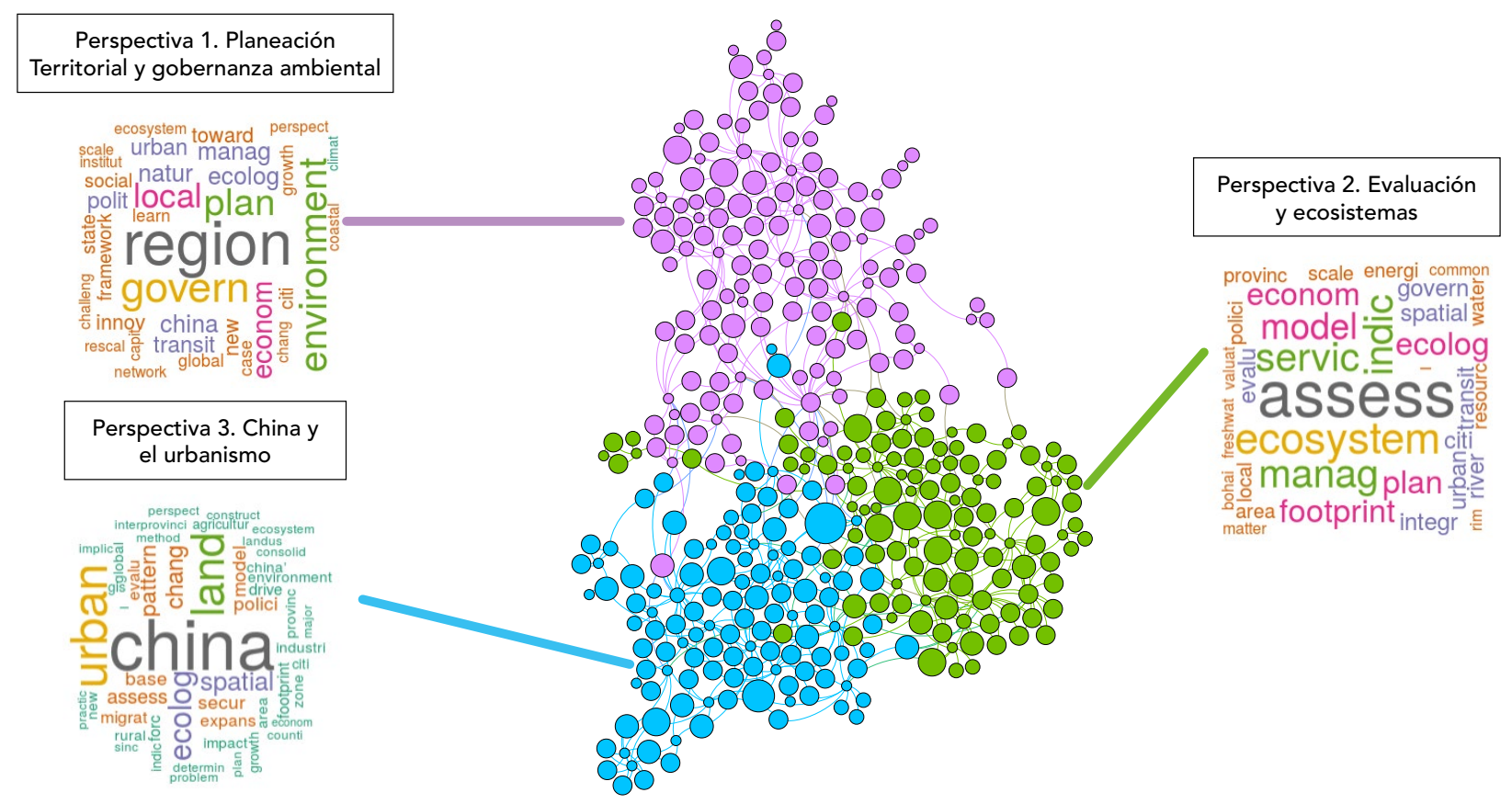

Figura 4. Red del desarrollo regional y la sostenibilidad

Fuente: elaboración de los autores (2020).

\section{Análisis de la red}

Tenemos una red conformada por 756 documentos que se obtuvieron de la base de datos Web of Science con parámetros de la ecuación establecida en la tabla 1. Utilizando el aplicativo Gephi, se generan los indicadores indegree (veces que un documento ha sido citado por otros), outdegree (veces que un nodo específico referencia a otros) y betweenness (este indicador muestra que el documento referencia ha sido citado por los demás para probar su importancia y aceptación en el tema de estudio).

Se realiza una revisión bibliográfica con los documentos más importantes de cada segmento previstos en la metodología del árbol (tabla 7); para la primera denominada raíz se seleccionaron 10 publicaciones (alto indegree), con los documentos hegemónicos o también llamados clásicos; para la segunda, llamada tronco (alto betweenness), se eligieron 10 documentos conocidos como estructurales; y para la tercera, nombrada como hojas (alto outdegree), se escogieron 30 documentos más recientes. 
Tabla 7. Árbol de desarrollo regional y sostenibilidad

\begin{tabular}{|c|c|c|c|c|c|c|}
\hline \multirow{6}{*}{ 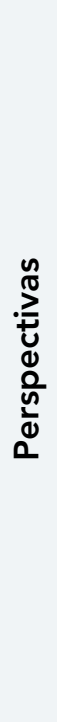 } & \multicolumn{2}{|c|}{ Cluster 1} & \multicolumn{2}{|c|}{ Cluster 2} & \multicolumn{2}{|c|}{ Cluster 3} \\
\hline & $\begin{array}{l}\text { Kollár } \\
\text { (2019) }\end{array}$ & $\begin{array}{c}\text { Bateman et al. } \\
\qquad(2013)\end{array}$ & $\begin{array}{l}\text { Rahma et al. } \\
\qquad(2019)\end{array}$ & $\begin{array}{c}\text { Doukas et al. } \\
\text { (2012) }\end{array}$ & $\begin{array}{l}\text { Liu et al. } \\
\text { (2019) }\end{array}$ & $\begin{array}{l}\text { Liu et al. } \\
\text { (2014) }\end{array}$ \\
\hline & $\begin{array}{c}\text { Ding et al. } \\
\text { (2019) }\end{array}$ & $\begin{array}{l}\text { Liu et al. } \\
\text { (2008) }\end{array}$ & $\begin{array}{l}\text { Wang et al. } \\
\qquad(2019)\end{array}$ & $\begin{array}{c}\text { Kajikawa et al. } \\
\text { (2008) }\end{array}$ & $\begin{array}{c}\text { Wang et al. } \\
\text { (2019) }\end{array}$ & $\begin{array}{c}\text { Wang et al. } \\
\text { (2013) }\end{array}$ \\
\hline & $\begin{array}{c}\text { Ding et al. } \\
\text { (2019) }\end{array}$ & $\begin{array}{c}\text { Von } \\
\text { Malmborg } \\
(2007)\end{array}$ & $\begin{array}{c}\text { Godlewska y } \\
\text { Sidorczuk-Pietraszko } \\
\text { (2019) }\end{array}$ & Mayer (2008) & $\begin{array}{l}\text { Li y Liu } \\
\text { (2019) }\end{array}$ & $\begin{array}{l}\text { Su et al. } \\
\text { (2011) }\end{array}$ \\
\hline & $\begin{array}{l}\text { Pallagst et al. } \\
\qquad(2019)\end{array}$ & Dühr (2005) & $\begin{array}{l}\text { Moscow State } \\
\text { Lomonosov } \\
\text { University y } \\
\text { Tambovtsev } \\
\text { (2019) }\end{array}$ & $\begin{array}{c}\text { Ness et al. } \\
(2007)\end{array}$ & $\begin{array}{l}\text { Li et al. } \\
\text { (2019) }\end{array}$ & $\begin{array}{l}\text { Fang y Lin } \\
\text { (2009) }\end{array}$ \\
\hline & $\begin{array}{l}\text { Gibbs y } \\
\text { O'Neill } \\
(2017)\end{array}$ & $\begin{array}{l}\text { Swyngedouw } \\
(2004)\end{array}$ & $\begin{array}{c}\text { Jiang et al. } \\
\quad(2018)\end{array}$ & $\begin{array}{l}\text { Haughton y } \\
\text { Counsell } \\
\text { (2004) }\end{array}$ & $\begin{array}{l}\text { Xuy } \\
\text { Chi } \\
\text { (2019) }\end{array}$ & $\begin{array}{l}\text { Fan y Li } \\
\text { (2009) }\end{array}$ \\
\hline \multirow{5}{*}{$\begin{array}{l}\text { 은 } \\
\text { 은 }\end{array}$} & \multicolumn{3}{|c|}{ Gibbs et al. (2005) } & \multicolumn{3}{|c|}{ Horlings y Marsden (2014) } \\
\hline & \multicolumn{3}{|c|}{ Wheeler (2009) } & \multicolumn{3}{|c|}{ Scott et al. (2013) } \\
\hline & \multicolumn{3}{|c|}{ Zavadskas y Turskis (2011) } & \multicolumn{3}{|c|}{ Shaker (2015) } \\
\hline & \multicolumn{3}{|c|}{ Boons et al. (2011) } & \multicolumn{3}{|c|}{ Shaker y Sirodoev (2016) } \\
\hline & \multicolumn{3}{|c|}{ Balezentis y Balezentis (2011) } & \multicolumn{3}{|c|}{ Tan y Lu (2016) } \\
\hline \multirow{5}{*}{$\frac{N}{\sqrt[N]{\alpha}}$} & \multicolumn{3}{|c|}{ Rees (1992) } & \multicolumn{3}{|c|}{ Chertow et al. (2008) } \\
\hline & \multicolumn{3}{|c|}{ WCED-World (1987) } & \multicolumn{3}{|c|}{ Singh et al. (2012) } \\
\hline & \multicolumn{3}{|c|}{ Costanza et al. (1997) } & \multicolumn{3}{|c|}{ Brauers (2010) } \\
\hline & \multicolumn{3}{|c|}{ Chertow (2000) } & \multicolumn{3}{|c|}{ Brauers et al. (2010) } \\
\hline & \multicolumn{3}{|c|}{ Kates et al. (2001) } & \multicolumn{3}{|c|}{ Brauers y Ginevičius (2009) } \\
\hline
\end{tabular}

Fuente: elaboración de los autores (2020).

\section{Documentos clásicos (raíz)}

Las bases teóricas están respaldadas en los documentos denominados hegemónicos o también llamados clásicos que fueron seleccionados y que sustentan sus aportes en un compromiso recíproco de cooperación de los países para asegurar la sostenibilidad ambiental del planeta a un largo plazo, sin afectar el desarrollo económico que permita satisfacer la demanda social. 
En 1987, la World Commission on Environment \& Development (n. d.) utiliza por primera vez el término de desarrollo sostenible en el contexto de la literatura, definiéndolo como "la satisfacción de las necesidades del ahora, sin afectar el mañana para las generaciones futuras". Se plantea por parte de la Comisión Mundial del Medio Ambiente la necesidad de que los países se ayuden entre sí para generar propuestas de solución a las amenazas que se perciban de modificación del medio ambiente a un largo plazo y traza una nueva perspectiva de interrelación de personas, recursos y medio ambiente en la generación de bienes y servicios como un principio común para atender la demanda social.

Más tarde, Rees (1992) hace referencia a dos términos que no se tenían presentes en los desarrollos de la economía urbana, como son las huellas ecológicas y la capacidad de carga. A partir de estos dos conceptos abre el debate y la discusión a la revisión de la sostenibilidad de la expansión económica de las ciudades con una mirada al cambio global.

Después, Costanza et al. (1997) llevaron a cabo un estudio en el cual plantearon unos parámetros de medición iniciales para cuantificar el valor de los servicios ambientales y una valoración de estos en el contexto del ecosistema global, permitiendo establecer igualmente un marco de posteriores estudios sobre este tema. La investigación efectuada contribuyó a sopesar el beneficio de los procesos de producción de bienes y servicios con relación a la afectación del ecosistema y la toma de decisión que implica este en el bienestar general del planeta.

A comienzo de este nuevo milenio, Chertow (2000) hace una publicación señalando la simbiosis industrial como una oportunidad potencial de mejora del medio ambiente, toda vez que la reutilización de productos por parte de otras empresas genera menos contaminación y, al mismo tiempo, los desechos se utilizan en otro sector de la industria en que se requiera para suplir diferentes mercados. En esta misma década, Chertow et al. (2008) revisan la relación de la simbiosis industrial y la teoría de la economía de aglomeraciones en los beneficios ambientales; allí, relacionan directamente la teoría de la economía de expansión con la simbiosis industrial, toda vez que la ubicación de dos o más empresas en un mismo espacio geográfico puede disminuir los impactos negativos al desarrollo sostenible del ecosistema y generar mayor eficiencia de su productividad. 
Por otro lado, se aprecia que la ciencia de la sostenibilidad fue presentada por Kates et al. (2001) como una ciencia para integrar los conocimientos científicos tradicionales y las diferenciaciones sectoriales entre las acciones de las personas que requerían estar en conexión con la agricultura, el transporte, la producción de energía y la vivienda, e infieren tres líneas de apoyo desde el conocimiento científico y de tecnologías para satisfacer el requerimiento de las personas, conservar el medio ambiente y generar cultura sostenible de consumo humano. Más adelante, Singh et al. (2012) describen varias metodologías para medir la sostenibilidad, las cuales convergen para indicar que el índice resultante se construye utilizando tres momentos importantes: normalización, ponderación y agregación.

Por último, se registran tres publicaciones que guardan relación con la medición de la sostenibilidad. En primer lugar, tenemos a Brauers y Ginevičius (2010) para medir la economía de las regiones belgas, que utilizan el método multimoora, el cual mezcla tres métodos de optimización multiobjetivo que chequean entre ellos, como el punto de referencia, los sistemas de razón y la forma multiplicativa completa. El segundo documento es presentado por Brauers et al. (2010) para exponer el desarrollo regional en Lituania, este usó el método moora, con fundamento en múltiples objetivos, que permite valorar el bienestar de la persona con respecto a la riqueza material, la educación, la salud, los diferentes ambientes de seguridad y el medio ambiente. Y, finalmente, Brauers y Ginevičius (2009) emplearon la descripción de robustez en la aplicación de las teorías económicas y el término de error en una ecuación lineal, que no solo se profundizó, sino que, además, se asimiló al significado de lenguaje común: de un cardinal a uno cualitativo.

\section{Documentos estructurales (tronco)}

Los documentos estructurales son aquellos que vinculan los documentos más recientes con los hegemónicos. Tienen la mayor centralidad, lo que significa que son los más citados por hojas y, al mismo tiempo, hacen referencia a la raíz. Estos documentos conectan las raíces del árbol con la red.

Un primer artículo publicado por Gibbs et al. (2005) indaga sobre los problemas que dificultan la conformación de redes, como también los contratiempos para producir confianza y generar apoyo colaborativo entre empresas en el desarrollo de proyectos de producción que preserven el ambiente; y se interrogan sobre el actuar de los encargados 
de diseñar y promover las políticas públicas de desarrollo industrial. Posteriormente, en esta línea, Boons et al. (2011) resaltan el auge de la simbiosis industrial promovida en todo el mundo por las agencias del gobierno, por los organismos no gubernamentales y por las mismas empresas privadas, y producen toda una información teórica que permite analizar el proceso que conlleva que los sistemas industriales regionales modifiquen su producción en la solución para disminuir el impacto ambiental.

Para Brauers et al. (2010) y Wheeler (2009), planificar la conservación ambiental regional no es una tarea fácil y el aumento de preferencia en las regiones de urbanizar debe examinarse con mucha prevención, por lo que desarrollan una mirada al futuro de la planificación para las regiones en un marco de sostenibilidad y generan una alarma en los debates sobre el apresurado desarrollo regional, teniendo como referente que el crecimiento en las regiones hace más difícil la conservación del ecosistema que un incremento orientado localmente.

Luego, Boons et al. (2011) y Zavadskas y Turskis (2011) se dieron a la tarea de revisar qué métodos recientes se estaban utilizando en el análisis de comparación y en la valoración de alternativas para medir el desarrollo económico de una región, encontrando que los métodos de valoración de criterios múltiples son usados generalmente porque se desprenden de factores subjetivos y por la facilidad para conocer las dificultades marcadas por diferentes intereses del problema. En este mismo tiempo, Balezentis y Balezentis (2011) innovan en la Unión Europea con el uso del método de toma de decisiones multicriterio en la formulación de los programas y políticas públicas de desarrollo regional.

Posteriormente, Horlings y Marsden (2014) y Scott et al. (2013) calificaron las franjas rurales urbanas como aquellos lugares donde converge la ruralidad de las regiones con las ciudades, espacios sin una atención orientada para la conservación ambiental por derecho propio, enumeraron el alcance, la naturaleza y las razones que llevaron al colapso de las políticas internas de las franjas rurales urbanas, y prestaron especial atención a varios aspectos del enfoque por ecosistemas y el marco de planificación espacial, lo que refleja la enorme diferencia entre el entorno natural y el entorno construido.

Según Horlings y Marsden (2014), el cambio de desarrollo se ve reorientado progresivamente de una producción basada en la agricultura a un desarrollo que recoge aspectos 
enfocados al lugar. Este nuevo paradigma de desarrollo rural en Europa es inferido por tres aspectos muy importantes: la innovación, las nuevas interfaces y la reorientación de los recursos del territorio. Luego, Horlings y Marsden (2014), Scott et al. (2013) y Richard Ross Shaker (2015) se enfocan en determinar y analizar el grado de interconexión entre una serie de indicadores de sostenibilidad y si se puede simplificar en factores de desarrollo básicos clave para comprender mejor el desarrollo sostenible, logrando con la investigación aclarar muchos aspectos de la relación humano-ambiental que influye en el desarrollo sostenible de la humanidad.

En las últimas revisiones, se encuentran las realizadas por Shaker y Sirodoev (2016), cuyo propósito era generar un índice milimétrico local para reflejar los tres aspectos principales del desarrollo sostenible de Moldavia; evaluar cuantitativamente la interrelación de las submedidas utilizadas para crear un índice local integral de desarrollo sostenible; y visualizar y explicar el patrón espacial del desarrollo sostenible.

Para concluir, Tan y Lu (2016), teniendo en cuenta las deficiencias de los modelos tradicionales y la naturaleza multidimensional de los problemas de sostenibilidad regional, propusieron un modelo integrado de análisis de componentes principales no lineal y ortonormalización de Gram-Schmidt, y otros métodos para evaluar el desarrollo sostenible regional desde tres perspectivas: los sistemas sociales, económicos y medioambientales.

\section{Perspectivas}

Esta sección incluye algunas publicaciones que permiten reconocer la interrelación entre el pasado y el presente para comprender las prácticas, tendencias y problemas en él, de manera que se pueda ver intuitivamente el futuro y comprender el pasado. A continuación, se sintetizan los principales hallazgos y futuras líneas de investigación respecto al desarrollo regional y la sostenibilidad, por medio del análisis de tres perspectivas (tabla 8) identificadas en los documentos recientes: 1) planeación territorial y gobernanza ambiental; 2) evaluación y ecosistemas; y 3) China y el urbanismo. 


\section{Perspectiva 1: planeación territorial y gobernanza ambiental}

En esta perspectiva, las publicaciones que se han realizado enfocan su mirada hacia el papel de las autoridades locales en cuanto a la regulación del uso de las diversas áreas de producción económica y a las acciones para evitar el deterioro de los ecosistemas.

Inicialmente, Swyngedouw (2004) analizó los cambios en la escala del Estado y el surgimiento de nuevas conexiones entre la escala de gobernanza. A su vez, redefinió la relación entre el Estado/gobernanza y la sociedad civil o entre el poder del Estado y los ciudadanos. Complementa que los efectos destructivos de los procesos de localización global indican que el espacio para la circulación del capital se ha incrementado, mientras que el ajuste de la relación producción-consumo ha disminuido, cambiando el equilibrio de poder de una manera polarizada importante. Después, Dühr (2005) revisa el papel de las autoridades locales en una red de partes interesadas relacionadas con el desarrollo sostenible regional y explora su potencial para estimular el aprendizaje y la innovación asociados con el desarrollo sostenible.

Para abordar las devastadoras crisis ambientales y mejorar el bienestar humano, China ha estado implementando una serie de políticas nacionales sobre pagos por servicios ambientales. Dos de ellos, el Programa de Conservación de Bosques Naturales y el Programa de Devolución de Tierras Agrícolas a los Bosques, se encuentran entre los más grandes del mundo debido a sus ambiciosos objetivos, escalas masivas, enormes pagos e impactos potencialmente enormes (Liu et al., 2008). En países como el Reino Unido, incorporaron los servicios de los ecosistemas a la toma de decisiones económicas teniendo en cuenta el impacto del cambio climático, utilizaron modelos espaciales explícitos y métodos de valoración para estimar el valor económico comparable de estos servicios (Bateman et al., 2013).

Luego, Gibbs y O’Neill (2017) abordan las necesidades del deterioro ambiental y el cambio global relacionadas con el desarrollo económico, con el fin de estimular a la comunidad de investigación regional a responder con mayor entusiasmo a la transformación sostenible. Para Ding et al. (2019), el pago por servicios ecológicos es reconocido como el nivel más eficaz para promover el desarrollo sostenible. Sin embargo, es más probable que las regiones de los países en desarrollo y las economías emergentes caigan en un círculo 
vicioso. Los gobiernos locales y las comunidades piden con entusiasmo mejoras en las condiciones sociales y económicas a expensas del deterioro y el menoscabo ambiental.

Para Pallagst et al. (2019), en el contexto del desarrollo urbano se han desarrollado zonas verdes de innovación para promover soluciones en áreas desatendidas.

Los tipos de usos potenciales en estas áreas no están predeterminados, pero deben ser experimentales e innovadores. La revisión literaria (Ding et al., 2019) indica que se hace necesario introducir un modelo de gobierno basado en el valor del servicio para identificar las deficiencias de la gobernanza ambiental regional existente y establecer planes innovadores y agendas de transición para innovar y reorganizar las instituciones regionales y cambiar el papel de los grupos y organizaciones de actores regionales. Por último, Némediné Kollár et al. (2020) argumentan que la creación de ciudades inteligentes es importante como proyecto insignia de desarrollo, porque pueden estimular la innovación, el crecimiento, la prosperidad y el bienestar en el país o, incluso, en la región.

Las líneas de investigación futuras, para Ding et al. (2019), pueden centrarse en la integración de mecanismos de planificación y comercialización más completos, y explorar el impacto de la transición geológica en la gobernanza ambiental de la región en los países en desarrollo. Para Gibbs y O'Neill (2017), igualmente se necesita analizar el entorno específico (ubicación) en el que la transición se fusiona y se desarrolla, prestando atención a las conexiones e interacciones geográficas (es decir, relaciones espaciales) dentro y entre las transiciones, y lugares. Además, para Bateman et al. (2013), es importante que los trabajos siguientes puedan indagar por los cambios temporales y espaciales de los servicios de los ecosistemas, así como por los efectos sinérgicos, como el cambio climático y el uso de la tierra.

\section{Perspectiva 2: evaluación y ecosistemas}

En este compendio de publicaciones se aborda el concepto de ecosistemas y se mencionan algunas herramientas y resultados en su aplicabilidad en la evaluación de sostenibilidad. Para Haughton y Counsell (2004), cualquier análisis de la estrategia regional de desarrollo sostenible de un país debe basarse en la comprensión de las estrategias elegidas por el país en diferentes escalas y capacidades de gobernanza. Estas estrategias incluyen formas de 
reutilizar el poder, los recursos y la relación entre los participantes en el nuevo sistema de gobernanza.

Ness et al. (2007) proporcionan una clasificación de las herramientas de evaluación de la sostenibilidad con el objetivo de ampliar la comprensión de la evaluación de la sostenibilidad de un campo centrado en el medio ambiente a una interpretación más extensa de la sostenibilidad. En su momento, Kajikawa (2008) adelantó un debate sobre la conexión entre la ciencia del desarrollo sostenible y otras disciplinas científicas, y si la ciencia del desarrollo sostenible debería etiquetarse como una disciplina diferente. Concluyó que algunas personas pueden incluso ver la ciencia del desarrollo sostenible como una agenda política, oculta como un campo de investigación científica. Sin embargo, en comparación con tales discusiones, es más importante realizar un trabajo científico sobre temas de sostenibilidad y cumplimiento de dichos temas.

Por su parte, Mayer (2008) manifestó que la sostenibilidad estaba cambiando rápidamente de un concepto abstracto a un estado mensurable de ecosistemas humanos dinámicos. La gran cantidad de indicadores económicos, sociales y ambientales actualmente disponibles brindan una visión incomprensible de la sostenibilidad del sistema. Por lo tanto, para ayudar a la toma de decisiones, estos indicadores se proponen en el contexto de un marco conceptual, o se agregan cuantitativamente en el índice. Continuando con los aportes, Doukas et al. (2012) efectuaron el análisis de componentes principales (PCA) basado en los resultados del European Energy Star en las siguientes dos regiones: comunidades agrícolas y de montaña, permitiendo desarrollar la personalización apropiada de PCA para agregar indicadores de sostenibilidad a fin de capturar interacciones e interdependencias relacionadas. Posteriormente, Jiang et al. (2018) enriquecieron los estudios con un sistema de índice de evaluación integral de tres niveles para nichos naturales, económicos y sociales, incluidos niveles de objetivo, subjetivo, e índice para evaluar el nicho de recursos provinciales, medio ambiente, subsistemas económicos y sociales, y la ecología general del ecosistema.

Recientemente, Rahma et al. (2019) dieron a conocer el nuevo índice integral incorporando factores económicos, sociales y ambientales en los indicadores regionales de desarrollo sostenible. A su vez, Wang et al. (2019) propusieron que comprender el verdadero valor de los servicios de los ecosistemas puede promover el mantenimiento y la conser- 
vación de los servicios de los ecosistemas regionales y proporcionar una base científica para formular planes regionales para la construcción de una civilización ecológica, planes de desarrollo sostenible y políticas de compensación ecológica.

Al mismo tiempo, Godlewska y Sidorczuk-Pietraszko (2019) propusieron un índice integral, es decir, un índice que "agrega un conjunto de índices individuales basado en el modelo básico del concepto multidimensional que se va a medir". Para concluir esta mirada, nos encontramos a Tambovtsev (2019), quien manifiesta que el cambio al desarrollo sostenible necesita no solo del trabajo arduo de los gobiernos, sino también de la acción de muchos microactores, empresas, familias e individuos. Diversas investigaciones han demostrado que las instituciones desempeñan un papel importante en la transición al desarrollo sostenible. Igualmente, pueden simplificar el proceso de transición y ralentizarlo, por ejemplo, agudizando las condiciones para expandir el proceso de innovación, especialmente el espíritu empresarial 'verde'.

Es esencial seguir realizando investigaciones en diferentes aspectos de evaluación y ecosistemas, sin embargo, Rahma et al. (2019) recomiendan algunos temas de relevancia como analizar el impacto de los desastres naturales en el desarrollo regional o la dinámica de las situaciones políticas regionales. Así mismo, Tambovtsev (2019) propone determinar la composición del entorno institucional regional para el desarrollo sostenible en las distintas regiones, resolver tareas desajustadas en el marco de la transición al desarrollo sostenible e identificar y analizar los temas de transformación institucional regional formal que restringen la participación de los actores en la solución. Como también, enuncian Jiang et al. (2018), la de llevar a cabo estudios que establezcan indicadores directamente relacionados con las políticas y la cultura ecológicas, e incorporarlos al sistema de evaluación de manera más integral con el nivel ecológico.

\section{Perspectiva 3: China y el urbanismo}

En esta perspectiva las publicaciones que se abordaron se refirieron a conocer y examinar las características temporales y espaciales de los cambios en la intensidad del uso de la tierra en un país con un área relativamente grande y condiciones locales complejas como lo es China. 
Para Fang y Lin (2009) y Fan y Li (2009), la delimitación de áreas orientada a funciones principales es una guía para optimizar el patrón espacial del desarrollo regional de China, que contiene inherentemente la innovación teórica y metodológica en el campo de la geografía económica. Así mismo, Fang y Lin (2009) consideran que existe una relación lineal positiva entre urbanización y huella ecológica, una relación lineal negativa entre urbanización e intensidad de huella ecológica, una relación lineal negativa entre déficit ecológico y excedente, y una relación exponencial negativa entre urbanización e indicadores de calidad ambiental ecológicos integrales.

Más tarde, Su et al. (2011) presentan su propuesta de investigación para medir la seguridad ecológica de la Tierra, que consiste en diseñar un método para cuantificar el estado de los ecosistemas terrestres a lo largo del tiempo.

Del mismo modo, Wang et al. (2013) dan a conocer una investigación que propone un sistema completo de indicadores ambientales mediante el establecimiento de un cuadro de mando integral y un modelo de PSR, y utiliza los indicadores definidos y los métodos de análisis de componentes principales para evaluar la urbanización y la sostenibilidad ambiental. En esta línea, Liu et al. (2014) expusieron el método de regionalización dinámica para analizar el modelo espacial del límite de división geográfica, las características internas de la región y los cambios en el crecimiento y declive de las unidades.

Consecuentemente, Liu et al. (2019) plantean el método de zonificación de retribución del desarrollo regional desde el criterio de la eficiencia y sostenibilidad del uso del suelo, y proponen la intensidad y eficiencia de tipos potenciales, así como los objetivos de desarrollo espacial de diferentes zonas de equilibrio de crecimiento y área terrestre. De igual forma, Li y Liu (2019) establecieron un sistema de indicadores para China y sus antecedentes de urbanización, y propusieron un método de entropía mejorada para evaluar las dimensiones sociales, económicas y ambientales del desempeño de la urbanización nacional y provincial de China. De manera semejante, Li et al. (2019) presentaron su estudio de diseño de un patrón de seguridad ecológica regional que puede moderar eficazmente las contradicciones causadas por la rápida urbanización y el uso insensato del suelo, y proporcionar una forma importante de asegurar el desarrollo regional sostenible. 
Concluyendo esta revisión literaria, se encuentra, por un lado, a Xu y Chi (2019), que amplían el concepto de magnitud del uso de la tierra de una manera más completa para explicar todos los tipos de uso de esta y facilitando otra forma de vincular dicho uso y el desarrollo regional, y considerando la heterogeneidad espacial y la no estacionariedad de las variables como factores que impulsan el cambio de uso del suelo en China; y, por otra parte, a Wang et al. (2019), exponiendo que la sustitución de suelos de construcción urbano-rural ha logrado una serie de metas como la velocidad de desarrollo de la agricultura, modelos nuevos de construcción de infraestructura rural, aumentando las condiciones de vida, liberando los recursos de la tierra rural y ampliando los cultivos de tierra, facilitando así buenos antecedentes para otras áreas de programas experimentales y el trabajo futuro.

Esta perspectiva requiere extender la investigación actual sobre la evaluación de la urbanización sostenible a las predicciones futuras y al análisis de escenarios. Por otro lado, para Wang et al. (2019), es importante realizar estudios que permitan orientar la asignación de recursos y el uso óptimo de los recursos terrestres en la orientación de planificación a escala macrorregional, y diseñar una medida integral de eficiencia de la tierra (Li et al., 2019). El umbral de área óptima de fuentes ecológicas sigue siendo un tema clave para la investigación futura sobre la construcción de patrones de seguridad ecológica (Xu \& Chi, 2019). Los aspectos temporales y espaciales de los mecanismos que impulsan el cambio de uso de la tierra no se han explorado completamente.

\section{Conclusiones y limitaciones}

El interés de la sociedad científica por este campo del conocimiento ha aumentado, reflejando su tasa de crecimiento anual del 17.39\%, y la producción científica de los últimos cinco años representó el $52.53 \%$ del total de publicaciones. Según la información obtenida en WoS, el autor con más publicaciones fue Fan Jie; por otro lado, el autor más citado es David Gibbs. El país con mayor número de publicaciones sobre este tema es China, que muestra el $22.5 \%$ de toda la información analizada. Rumania y Rusia ocuparon el segundo y tercer lugar, representando el $8.1 \%$ y el $7.0 \%$ de la producción científica mundial, respectivamente. La red de cooperación entre países muestra que el grupo principal está 
compuesto por China, Estados Unidos y Japón. El grupo se destaca por estar compuesto por países de dos continentes diferentes (América y Asia), lo que implica una conexión importante entre la escuela y diferentes tendencias académicas.

La base de conocimientos científicos relacionados con el desarrollo regional y la sostenibilidad se apoyó inicialmente en los documentos hegemónicos o también conocidos como documentos clásicos, centran sus trabajos en la necesidad de los países de trabajar en cooperación para asegurar la sostenibilidad del medio ambiente terrestre. A largo plazo, el aporte no afectará el desarrollo económico que permita satisfacer las necesidades sociales. Posteriormente, durante la revisión de los documentos estructurados miraban la planificación futura del área en el marco de la sustentabilidad, y emitieron una alarma en el debate sobre el descuidado desarrollo regional. Tomando esto como referencia, se observa que el crecimiento de la región hace que la protección del ecosistema sea más difícil si no existe un incremento orientado localmente.

El estudio de las perspectivas permitió reconocer la interrelación entre el pasado y el presente para comprender las prácticas, tendencias y problemas en él, de modo que intuitivamente se pueda ver el futuro y comprender el pasado. Los principales hallazgos y orientaciones futuras de la investigación sobre el desarrollo regional y la sostenibilidad se enfocan en tres puntos de vista: 1) planificación territorial y gobernanza ambiental, 2) evaluación y ecosistemas, y 3) China y urbanismo.

En la primera óptica, se observa que las publicaciones se centran en el papel de las autoridades locales en la regulación del uso de diversos campos de producción. La segunda mirada incluye una serie de bosquejos de publicaciones que discuten el concepto de ecosistemas y mencionan algunas herramientas y resultados para ilustrar su aplicabilidad en la evaluación de la sostenibilidad. Y, por último, se identifica que, desde esta perspectiva, la publicación involucrada se refiere a comprender y estudiar las características temporales y espaciales de los cambios en la intensidad del uso del suelo en un país con una gran superficie y condiciones locales complejas (como China).

Para finalizar, y teniendo como antecedente la revisión bibliográfica, se plantea la necesidad de explorar más a fondo cómo se producen las transformaciones sociales que 
tienen como objetivo el desarrollo sostenible, cómo pueden ser apoyados por diversos medios políticos y qué opciones necesita la sociedad para estas transformaciones.

La utilización de solo la base de datos de búsqueda de información WoS puede generar una limitación y posible sesgo sobre la revisión efectuada acerca del desarrollo regional y la sostenibilidad.

\section{Futuras investigaciones}

Las direcciones de investigación futuras pueden centrarse en focalizar estudios hacia países del continente americano, como también a complementar la agenda propuesta por los autores en la revisión realizada. Algunas líneas son: proponer la integración de mecanismos más completos de planificación y comercialización y discutir el impacto de la transición geológica en la gobernanza ambiental regional en los países en desarrollo. También es necesario analizar el entorno específico (ubicación) en el que la transición se fusiona y se desarrolla; y prestar atención a la transición, las conexiones geográficas y las interacciones (es decir, relaciones espaciales) entre la ubicación y otros lugares y entre ellos; además, investigar los cambios temporales y espaciales en los servicios de los ecosistemas, y los efectos sinérgicos, como el cambio climático y el uso de la tierra.

Igualmente, es conveniente llevar a cabo estudios que establezcan indicadores directamente relacionados con las políticas y la cultura ecológicas e incorporarlos al sistema de evaluación para analizar de manera más integral el nivel ecológico. Así mismo, determinar la composición del entorno institucional regional para el desarrollo sostenible en las distintas regiones, resolver tareas desajustadas en el marco de la transición al desarrollo sostenible, e identificar y revisar los temas de transformación institucional regional formal que restringen la participación de los actores en la solución.

\section{Referencias}

Balaban, M., Župljanin, S. S., \& Nešović, D. (2019). Regional sustainability of local and rural development. Ekonomika Poljoprivrede, 66(4), 1173-1186. 
Balezentis, A., \& Balezentis, T. (2011). Framework of strategic management model for strategy Europe 2020: diachronic analysis and proposed guidelines. Engineering Economics, 22(3). https://doi.org/10.5755/j01.ee.22.3.517

Bateman, I. J., Harwood, A. R., Mace, G. M., Watson, R. T., Abson, D. J., ... Termansen, M. (2013). Bringing ecosystem services into economic decision making: land use in the United Kingdom. Science, 341(6141), 45-50.

Blondel, V. D., Guillaume, J.-L., Lambiotte, R., \& Lefebvre, E. (2008). Fast unfolding of communities in large networks. Journal of Statistical Mechanics: Theory and Experiment, (10), P10008. https://doi.org/10.1088/1742-5468/2008/10/p10008

Boons, F., Spekkink, W., \& Mouzakitis, Y. (2011). The dynamics of industrial symbiosis: a proposal for a conceptual framework based upon a comprehensive literature review. Journal of Cleaner Production, 19(9-10), 905-911.

Braam, R. R., Moed, H. F., \& Van Raan, A. F. J. (1991). Mapping of science by combined co-citation and word analysis. I. Structural aspects. Journal of the American Society for Information Science, 42(4), 233-251.

Brauers, W. K. M., \& Ginevićius, R. (2009). Robustness in regional development studies. The case of Lithuania. Journal of Business Economics and Management, 10(2), 121-140.

Brauers, W. K. M., \& Ginevićius, R. (2010). The economy of the Belgian regions tested with multimoora. Journal of Business Economics and Management, 11(2), 173-209.

Brauers, W. K. M., Ginevićius, R., \& Podvezko, V. (2010). Regional development in Lithuania considering multiple objectives by the moora method / Lietuvos regioninès plètros daugiaaspektis vertinimas moora metodu. Technological and Economic Development of Economy, 16(4), 613-640.

Buitrago, S., Duque, P., \& Robledo, S. (2020). Branding corporativo: una revisión bibliográfica. Económicas CUC, 41(1). https://doi.org/10.17981/econcuc.41.1.2020.org.1

Chertow, M. R. (2000). Industrial symbiosis: literature and taxonomy. Annual Review of Energy and the Environment, 25(1), 313-337.

Chertow, M. R., Ashton, W. S., \& Espinosa, J. C. (2008). Industrial symbiosis in Puerto Rico: environmentally related agglomeration economies. Regional Studies, 42(10), 1299-1312.

Choi, K. S., \& Labhsetwar, V. K. (2020). Sustainable agricultural growth for rural development in Asia: a review. Irrigation and Drainage. https://doi.org/10.1002/ird.2494

Cohn, A. S., Newton, P., Gil, J. D. B., Kuhl, L., Samberg, L., ... Northrop, S. (2017). Smallholder agriculture and climate change. Annual Review of Environment and Resources, 42(1), 347-375. 
Costanza, R., D’Arge, R., De Groot, R., Farber, S., Grasso, M., ... Van den Belt, M. (1997). The value of the world's ecosystem services and natural capital. Nature, 387(6630), 253-260.

Díez-Gómez, D. A., Guillén, M., \& Rodríguez, M. del P. (2019). Revisión de la literatura sobre la toma de decisiones éticas en organizaciones. Información Tecnológica, 30(3), 25-38.

Ding, Y., \& Cronin, B. (2011). Popular and/or prestigious? Measures of scholarly esteem. Information Processing \& Management, 47(1), 80-96. https://doi.org/10.1016/j. ipm.2010.01.002

Ding, X., Zhou, C., Mauerhofer, V., Zhong, W., \& Li, G. (2019). From environmental soundness to sustainable development: improving applicability of payment for ecosystem services scheme for diverting regional sustainability transition in developing countries. Sustainability: Science Practice and Policy, 11(2), 361.

Ding, X., Zhou, C., Zhong, W., \& Tang, P. (2019). Addressing uncertainty of environmental governance in environmentally sensitive areas in developing countries: a precise-strike and spatial-targeting adaptive governance framework. Sustainability: Science Practice and Policy, 11(16), 4510.

Donato, V. (2017). Towards design process validation integrating graph theory into BIM. Architectural Engineering and Design Management, 13(1), 22-38.

Donthu, N., Kumar, S., \& Pattnaik, D. (2020). Forty-five years of Journal of Business Research: a bibliometric analysis. Journal of Business Research, 109, 1-14.

Doukas, H., Papadopoulou, A., Savvakis, N., Tsoutsos, T., \& Psarras, J. (2012). Assessing energy sustainability of rural communities using principal component analysis. Renewable and Sustainable Energy Reviews, 16(4), 1949-1957.

Dühr, S. (2005). Spatial policies for regional sustainable development: a comparison of graphic and textual representations in regional plans in England and Germany. Regional Studies, 39(9), 1167-1182.

Duque, P., \& Cervantes-Cervantes, L.-S. (2019). Responsabilidad social universitaria: una revisión sistemática y análisis bibliométrico. Estudios Gerenciales, 451-464.

Duque, P., \& Duque, J. (2020). Marketing digital y comercio electrónico: un análisis bibliométrico. En M. I. Redondo Ramírez, A. M. Barrera Rodríguez \& C. C. Duque Gómez (Eds.), Nuevos modelos de negocio (pp. 74-96). Centro de Investigaciones, Facultad de Ciencias Económicas, Administrativas y Contables.

Duque, P., Meza, O., Zapata, G., \& Giraldo, J. (2021). Internacionalización de empresas latinas: evolución y tendencias. Económicas CUC, 42(1). https://doi.org/10.17981/econcuc.42.1.2021.org.1 
Duque, P., Samboni, V., Castro, M., Montoya, L. A., \& Montoya, I. A. (2020). Neuromarketing: Its current status and research perspectives. Estudios Gerenciales, 36(157). https://doi. org/10.18046/j.estger.2020.157.3890

Duque, P., Toro, A., Ramírez, D., \& Carvajal, M. (2020). Marketing viral: aplicación y tendencias. Clio América, 14(27). Recuperado de http://revistas.unimagdalena.edu.co/index. $\mathrm{php/clioamerica/article/view/3759}$

Fang, C., \& Lin, X. (2009). The eco-environmental guarantee for China's urbanization process. Journal of Geographical Sciences, 19(1), 95-106.

Fan, J., \& Li, P. (2009). The scientific foundation of major function oriented zoning in China. Journal of Geographical Sciences, 19(5), 515-531.

Ferguson, R. (2012). Learning analytics: drivers, developments and challenges. International Journal of Technology Enhanced Learning, 4(5/6), 304.

Freeman, L. C. (1977). A set of measures of centrality based on betweenness. Sociometry, $40(1), 35$.

Geneletti, D., La Rosa, D., Spyra, M., \& Cortinovis, C. (2017). A review of approaches and challenges for sustainable planning in urban peripheries. Landscape and Urban Planning, 165, 231-243.

Gentner, D. (1983). Structure-mapping: a theoretical framework for analogy*. Cognitive Science, $7(2), 155-170$.

Gibbs, D., Deutz, P., \& Proctor, A. (2005). Industrial ecology and eco industrial development: a potential paradigm for local and regional development? Regional Studies, 39(2), 171-183.

Gibbs, D., \& O'Neill, K. (2017). Future green economies and regional development: a research agenda. Regional Studies, 51(1), 161-173.

Godlewska, J., \& Sidorczuk-Pietraszko, E. (2019). Taxonomic assessment of transition to the green economy in Polish regions. Sustainability, 11(18), 5098. https://doi.org/10.3390/ su11185098

Haughton, G., \& Counsell, D. (2004). Regions and sustainable development: regional planning matters. The Geographical Journal, 170(2), 135-145.

Hjørland, B. (2013). Citation analysis: a social and dynamic approach to knowledge organization. Information Processing \& Management, 49(6), 1313-1325.

Horlings, L. G., \& Marsden, T. K. (2014). Exploring the "new rural paradigm" in Europe: eco-economic strategies as a counterforce to the global competitiveness agenda. European Urban and Regional Studies, 21(1), 4-20. 
Hughes, R. (2010). The figure of Ramon Llull (Raimundus Lullus) and the significance of the recent companion volume to his Latin works in the Corpus Christianorum series. Faventia, $32(1), 177-188$.

Jacomy, M., Venturini, T., Heymann, S., \& Bastian, M. (2014). ForceAtlas2, a continuous graph layout algorithm for handy network visualization designed for the Gephi software. PLOS ONE, 9(6), e98679.

Jiang, Y., Sun, S., Wang, Y., \& Zheng, S. (2018). Niche evolution of China's provincial social-economic-natural complex ecosystems, 2005-2015. Sustainability: Science Practice and Policy, 10(8), 2824.

Kajikawa, Y. (2008). Research core and framework of sustainability science. Sustainability Science, 3(2), 215-239.

Kates, R. W., Clark, W. C., Corell, R., Hall, J. M., Jaeger, C. C., .. Svedlin, U. (2001). Environment and development. Sustainability science. Science, 292(5517), 641-642.

Kim, D., \& Lim, U. (2017). Social enterprise as a catalyst for sustainable local and regional development. Sustainability: Science Practice and Policy, 9(8), 1427.

Levy, S. J. (2002). Revisiting the marketing domain. European Journal of Marketing, 36(3), 299-304.

Li, L., \& Liu, Y. (2019). Spatial-temporal patterns and driving forces of sustainable urbanization in China since 2000. Journal of Urban Planning and Development, 145(4), 05019014.

Li, S., Xiao, W., Zhao, Y., Xu, J., Da, H., \& Lv, X. (2019). Quantitative analysis of the ecological security pattern for regional sustainable development: case study of Chaohu Basin in Eastern China. Journal of Urban Planning and Development, 145(3), 04019009.

Liu, J., Jin, X., Xu, W., Fan, Y., Ren, J., ... Zhou, Y. (2019). Spatial coupling differentiation and development zoning trade-off of land space utilization efficiency in eastern China. Land Use Policy, 85, 310-327.

Liu, J., Kuang, W., Zhang, Z., Xu, X., Qin, Y., ... Chi, W. (2014). Spatiotemporal characteristics, patterns, and causes of land-use changes in China since the late 1980s. Journal of Geographical Sciences, 24(2), 195- 210.

Liu, J., Li, S., Ouyang, Z., Tam, C., \& Chen, X. (2008). Ecological and socioeconomic effects of China's policies for ecosystem services. Proceedings of the National Academy of Sciences of the United States of America, 105(28), 9477-9482.

Mathieu, B., Sebastien, H., \& Mathieu, J. (2009). Gephi: an open source software for exploring and manipulating networks. International AAAI Conference on Weblogs and Social Media. Available from https://gephi.org/users/publications/ 
Mayer, A. L. (2008). Strengths and weaknesses of common sustainability indices for multidimensional systems. Environment International, 34(2), 277-291.

Meier, F. (2020). Social network analysis as a tool for data analysis and visualization in information behaviour and interactive information retrieval research. Proceedings of the 2020 Conference on Human Information Interaction and Retrieval, 10, 477=480.

Némediné Kollár, K., Topa, Z., Káposzta, J., \& Borbás, D. P. (2020). Making rural cities smart--By upgrading existing cities or creating new ones? Studia Mundi-Economica, 7(4), 113-124.

Ness, B., Urbel-Piirsalu, E., Anderberg, S., \& Olsson, L. (2007). Categorising tools for sustainability assessment. Ecological Economics: The Journal of the International Society for Ecological Economics, 60(3), 498-508.

Ohri, A. (2012). R for business analytics. Springer Science \& Business Media.

Pallagst, K., Vargas-Hernández, J., \& Hammer, P. (2019). Green innovation areas. En route to sustainability for shrinking cities? Sustainability: Science Practice and Policy, 11(23), 6674.

Persson, O. (1994). The intellectual base and research fronts of JASIS 1986-1990. Journal of the American Society for Information Science, 45(1), 31-38.

Price, D. J. (1965). Networks of scientific papers. Science, 149(3683), 510-515.

Rahma, H., Fauzi, A., Juanda, B., \& Widjojanto, B. (2019). Development of a composite measure of regional sustainable development in Indonesia. Sustainability: Science Practice and Policy, 11(20), 5861.

Rees, W. E. (1992). Ecological footprints and appropriated carrying capacity: what urban economics leaves out. Environment and Urbanization, 4(2), 121-130.

Rinkinen, S., Oikarinen, T., \& Melkas, H. (2016). Social enterprises in regional innovation systems: a review of finnish regional strategies. European Planning Studies, 24(4), 723-741.

Robledo, S., Osorio, G., \& López, C. (2014). Networking en pequeña empresa: una revisión bibliográfica utilizando la teoría de grafos. Revista Vínculos, 11(2), 6-16.

Russo, T., Alfredo, K., \& Fisher, J. (2014). Sustainable water management in urban, agricultural, and natural systems. Water, 6(12), 3934-3956.

Salazar, D. F. T., Álvarez, J. M. R., Garcés, J. J. H., \& Duque, P. (2020). Riesgos en las microfinanzas: una revisión y análisis bibliométrico*. Interfaces, 3(2). Recuperado de http://www. unilibrecucuta.edu.co/ojs/index.php/ingenieria/article/view/484

Scott, A. J., Carter, C., Reed, M. R., Larkham, P., Adams, D., ... Coles, R. (2013). Disintegrated development at the rural-urban fringe: re-connecting spatial planning theory and practice. Progress in Planning, 83, 1-52. 
Secundo, G., Ndou, V., Vecchio, P. D., \& De Pascale, G. (2020). Sustainable development, intellectual capital and technology policies: a structured literature review and future research agenda. Technological Forecasting and Social Change, 153, 119917.

Servillo, L., Atkinson, R., \& Russo, A. P. (2012). Territorial attractiveness in Eu urban and spatial policy: a critical review and future research agenda. European Urban and Regional Studies, 19(4), 349-365.

Shafique, M. (2013). Thinking inside the box? Intellectual structure of the knowledge base of innovation research (1988-2008): intellectual structure of innovation research (1988-2008). Strategic Management Journal, 34(1), 62-93.

Shaker, R. R. (2015). The spatial distribution of development in Europe and its underlying sustainability correlations. Applied Geography, 63, 304-314.

Shaker, R. R., \& Sirodoev, I. G. (2016). Assessing sustainable development across Moldova using household and property composition indicators. Habitat International, 55, 192-204.

Singh, R. K., Murty, H. R., Gupta, S. K., \& Dikshit, A. K. (2012). An overview of sustainability assessment methodologies. Ecological Indicators, 15(1), 281-299.

Stoffelen, A. (2020). Where is the community in geoparks? A systematic literature review and call for attention to the societal embedding of geoparks. Area, 52(1), 97-104.

Su, S., Li, D., Yu, X., Zhang, Z., Zhang, Q., ... Wu, J. (2011). Assessing land ecological security in Shanghai (China) based on catastrophe theory. Stochastic Environmental Research and Risk Assessment: Research Journal, 25(6), 737-746.

Swyngedouw, E. (2004). Globalisation or "glocalisation"? Networks, territories and rescaling. Cambridge Review of International Affairs, 17(1), 25-48.

Tambovtsev, V. L. (2019). Sustainable regional development: actual directions of institutional analysis. Journal of Institutional Studies, 11(3), 104-118.

Tan, F., \& Lu, Z. (2016). Assessing regional sustainable development through an integration of nonlinear principal component analysis and Gram Schmidt orthogonalization. Ecological Indicators, 63, 71-81.

Wallis, W. D. (2007). A beginner's guide to graph theory. https://doi.org/10.1007/978-0-81764580-9

Wang, C., Li, X., Yu, H., \& Wang, Y. (2019). Tracing the spatial variation and value change of ecosystem services in Yellow River Delta, China. Ecological Indicators, 96, 270-277.

Wang, J., Li, Y., Wang, Q., \& Cheong, K. C. (2019). Urban-rural construction land replacement for more sustainable land use and regional development in China: policies and practices. Land, 8(11), 171. 
Wang, Q., Yuan, X., Zhang, J., Mu, R., Yang, H., \& Ma, C. (2013). Key evaluation framework for the impacts of urbanization on air environment. A case study. Ecological Indicators, 24, 266-272.

Wasserman, S., \& Faust, K. (1994). social network analysis: methods and applications. Cambridge University Press.

Wheeler, S. (2009). Regions, megaregions, and sustainability. Regional Studies, 43(6), 863-876.

World Commission on Environment; \& Development. (n. d.). Report of the World Commission on Environment and Development: Our common future. A/42/427 Annex - uN Documents: gathering a body of global agreements. Available from http://www.un- documents.net/ wced-ocf.htm

Xu, F., \& Chi, G. (2019). Spatiotemporal variations of land use intensity and its driving forces in China, 2000-2010. Regional Environmental Change, 19(8), 2583-2596.

Yang, S., Keller, F. B., \& Zheng, L. (2016). Social network analysis: methods and examples. SAGE Publications.

Zavadskas, E. K., \& Turskis, Z. (2011). Multiple criteria decision making (мсдм) methods in economics: an overview / Daugiatiksliai sprendimų prièmimo metodai ekonomikoje: apžvalga. Technological and Economic Development of Economy, 17(2), 397-427.

Zhang, J., \& Luo, Y. (2017). Degree centrality, betweenness centrality, and closeness centrality in social network. In Proceedings of the $20172^{\text {nd }}$ International Conference on Modelling, Simulation and Applied Mathematics (MSAM 2017). https://doi.org/10.2991/ msam-17.2017.68

Zupic, I., \& Čater, T. (2015). Bibliometric methods in management and organization. Organizational Research Methods, 18(3), 429-472. 